\title{
Design of a multi-electrode array to measure cardiac conductivities
}

\author{
Barbara M. Johnston ${ }^{1}$
}

(Received 24 October 2012; revised 26 April 2013)

\begin{abstract}
Accurate determination of cardiac tissue parameters is essential in bidomain models that simulate the electrical activity of the heart and thereby contribute to understanding cardiovascular disease. Recent experimental work indicated the need for six parameters, which measure electrical conductivity in two domains (extracellular and intracellular), along and across the cardiac fibres within a sheet and also between sheets. This is in contrast to the available experimentally determined conductivities, which are sets of four values, where it is assumed that conductivities across the fibres within a sheet and between the fibre sheets are equal. This study presents a mathematical model that incorporates six bidomain conductivities. It also discusses the design of a multi-electrode array and inversion method to retrieve these
\end{abstract}

http://journal. austms.org.au/ojs/index.php/ANZIAMJ/article/view/6278 gives this article, (c) Austral. Mathematical Soc. 2013. Published June 5, 2013, as part of the Proceedings of the 16th Biennial Computational Techniques and Applications Conference. ISSN 1446-8735. (Print two pages per sheet of paper.) Copies of this article must not be made otherwise available on the internet; instead link directly to this URL for this article. 
conductivities (as well as a value for fibre rotation between the sheets). The sensitivity of electrode spacing in the array design is investigated. Subject class: $92 \mathrm{C} 30$

Keywords: bidomain model, cardiac conductivity values, electrodes, simulation

\section{Contents}

1 Introduction

$\mathrm{C} 273$

2 Model

$\mathrm{C} 274$

2.1 Governing equations and boundary conditions . . . . . . C274

2.2 Solution technique and modelling parameters . . . . . . C276

3 Methods

$\mathrm{C} 277$

3.1 Previous method for retrieving conductivities . . . . . . . C C277

3.2 Sensitivity analysis . . . . . . . . . . . . . . C279

4 Results and discussion

C279

4.1 Sensitivity analysis on the original 2D array . . . . . . C279

4.2 Sensitivity analysis for a 3D array . . . . . . . . . C C280

4.2.1 Closely spaced electrode set in a 3D array . . . . . C280

4.2.2 Widely spaced electrode sets in a 3D array . . . . . C282

4.3 Array design . . . . . . . . . . . . . . . . C282

5 Conclusions

C284

References

C285 


\section{Introduction}

Studies that model various types of electrocardiographic phenomena are important in understanding aspects of cardiac behaviour, especially where experimental study is not feasible. However, determining cardiac conductivities for use in such models presents both experimental and computational challenges [4]. The use of the bidomain model [1,31] in such studies is well established, but due to measurement and interpretation issues, only three widely varying [25] sets of bidomain conductivities are available [5, 23, 24].

Many techniques for finding the bidomain conductivities are based on the so-called four electrode technique [26, 19], which uses four collinear equispaced electrodes. Current is applied to the outer pair of electrodes and measurements are made on the inner pair. This technique is used in two ways to obtain the four conductivities. In the first method, measurements are made with the array aligned in both the longitudinal and transverse directions, while also varying the distance between electrodes to be both less than and greater than the space constant of the tissue [19]. The second approach is to use electrode spacings of the order of the space constant and apply AC current with variable frequency [16].

More recent extensions of these measuring techniques often involve arrays of electrodes, where the design of the array is based on the four electrode technique $[17,2,20,21,22,9,13,12,14,6]$. An example of one of these designs is an array of plunge electrodes $[9,10,7,3]$ that demonstrated that porcine ventricular tissue is electrically orthotropic, with three distinct propagation directions. This result challenged the commonly made assumption $[19,13$, 20, 27, 29, 21, e.g.] that the cardiac conductivities in the direction normal to the fibres, but in the plane of the fibre sheet, are equal to those in the direction normal to the sheet. This led to the inclusion of three distinct propagation directions in a number of recent studies [8, 30, 15, 32] and some of these showed that simulations that include three, rather than two, distinct propagation directions much more closely match experimental results. Despite 
the use of some six bidomain conductivity datasets $[9,8,18]$, none of these are fully determined by experiments.

Previous work in this area $[13,11,12]$ showed that it is possible to accurately retrieve four cardiac conductivity values, as well as a value for fibre rotation, from simulated measurements of potential on the heart surface. These studies introduced a mathematical model, a solution technique and an inversion procedure, as well as a two layer multi-electrode array, and investigated the accuracy of measurements. The aim of the current study is to design a new array that would make measuring six conductivities possible.

Section 2 describes the model and solution technique, while Section 3 presents an array design that was previously shown to be able to retrieve intracellular and extracellular conductivities in the longitudinal and transverse directions. The sensitivity of the electrodes is investigated by measuring the changes in electrode potentials caused by changes in input conductivities. Results from this investigation are presented in Section 4 and are used to propose an array design that may be suitable for measuring not only the four conductivities mentioned above but also those in the normal direction. Conclusions about the types of electrodes that should be included in the design, along with a quick method for establishing the suitability of a particular design, are presented in the final section 5 .

\section{Model}

\subsection{Governing equations and boundary conditions}

In the model, cardiac ventricular tissue is represented by a block of tissue, $2 \mathrm{~cm} \times 2 \mathrm{~cm}$ in the $x$ and $y$ coordinate directions and $1 \mathrm{~cm}$ in the $z$ direction with the epicardium (outer surface of the heart) represented by the $x y$-plane. The endocardium (inner surface of the heart) is represented by the plane at $z=1$, and contacts a volume of blood which is in the range $z>1$ (Figure 1). 
Cardiac tissue is an electrically anisotropic structure, consisting of sheets containing parallel strands of cells. It is well known that it is much easier for electric current to flow along the fibres than across them. Many previous models used four conductivity values, allowing for variation in the intracellular ( $i$ ) and extracellular $(e)$ domains, and along $(l=$ longitudinal $)$ and transverse $(t)$ to the cardiac fibres. Although there are two directions transverse to the fibres (within the sheet $(\boldsymbol{t})$ and normal $(\mathfrak{n})$ to the sheet), it is often assumed, to make the problem more tractable, that conductivity in the two transverse directions is equal. The proposed model relaxes that condition, giving six bidomain electrical conductivities: $g_{i l}, g_{i t}, g_{i n}, g_{e l}, g_{e t}$ and $g_{e n}$.

The governing equations for the bidomain model [28] are

$$
\nabla \cdot M_{i} \nabla \phi_{i}=\frac{\beta}{R}\left(\phi_{i}-\phi_{e}\right) \text { and } \nabla \cdot M_{e} \nabla \phi_{e}=-\frac{\beta}{R}\left(\phi_{i}-\phi_{e}\right)-I_{s}
$$

where

$$
M_{p}(x, y, z)=\left[\begin{array}{ccc}
\left(g_{p l}-g_{p t}\right) c^{2}+g_{p t} & \left(g_{p l}-g_{p t}\right) c s & 0 \\
\left(g_{p l}-g_{p t}\right) c s & \left(g_{p l}-g_{p t}\right) s^{2}+g_{p t} & 0 \\
0 & 0 & g_{p n}
\end{array}\right]
$$

and $\phi_{e}$ is the extracellular potential, $\phi_{i}$ is the intracellular potential, $\beta$ is the surface to volume ratio of the cells, $R$ is the specific membrane resistance and $I_{s}$ is the external current source per unit volume applied in the extracellular space. The tensors $\mathbf{M}_{\mathrm{p}}$, for $p=i, e$, describe the anisotropic conductivity as well as linear fibre rotation from the epicardium to the endocardium through an angle $\alpha$ [13], with $\mathrm{c}=\cos \alpha z$ and $s=\sin \alpha z$.

The potential distribution in the blood, $\phi_{\mathrm{b}}$, is governed by Laplace's equation

$$
\nabla^{2} \phi_{\mathrm{b}}=0 \text {. }
$$

The boundary conditions are the same as the model of Johnston et al. [13]:

$$
\begin{aligned}
& \text { at } z=0, \quad \frac{\partial \phi_{e}}{\partial z}=\frac{\partial \phi_{\mathrm{i}}}{\partial z}=0 ; \\
& \text { at } z=1, \quad \phi_{e}=\phi_{\mathrm{b}}, \quad g_{\mathrm{b}} \frac{\partial \phi_{\mathrm{b}}}{\partial z}=g_{\mathrm{en}} \frac{\partial \phi_{\mathrm{e}}}{\partial z}, \quad \frac{\partial \phi_{\mathrm{i}}}{\partial z}=0,
\end{aligned}
$$



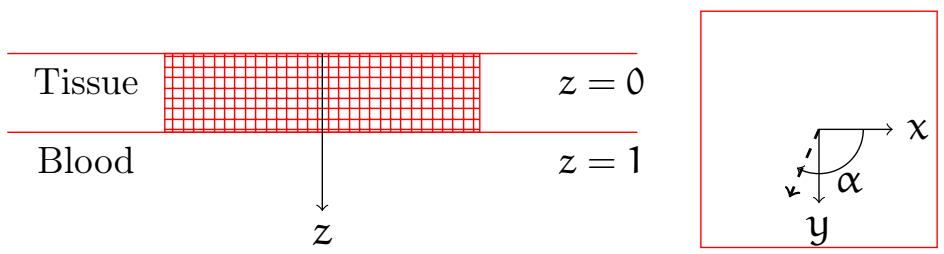

Figure 1: A schematic view of the tissue-blood model. The left panel is a cross-sectional view and the right panel is a plan view, looking from the negative $z$ direction, with the fibre direction on the epicardium along the $x$ axis.

where $g_{b}$ is the conductivity of blood. At the $\boldsymbol{x}$ and $\boldsymbol{y}$ boundaries, where $\mathfrak{n}$ is the outwardly pointing normal,

$$
\mathbf{M}_{e} \nabla \phi_{e} \cdot \mathfrak{n}=0, \quad \mathbf{M}_{i} \nabla \phi_{i} \cdot \mathfrak{n}=0 \quad \text { and } \quad \nabla \phi_{b} \cdot \mathfrak{n}=0 .
$$

\subsection{Solution technique and modelling parameters}

The solution technique for this model is very similar to that of the four conductivity model of Johnston et al. [13] and it involves the expansion of $\phi_{i}$ and $\phi_{e}$ as Fourier series, followed by a one dimensional finite difference scheme to find the series coefficients. This means that, given an applied potential, the potential at each individual point in the domain (for example, at an electrode) is obtained by summing the series at that point.

Two sets of conductivity values are used, the nominal four conductivity set used previously $[12,25]$ and the six conductivity set of Hooks et al. [8], shown in Table 1. Other parameters used are $\mathrm{g}_{\mathrm{b}}=6.7 \mathrm{mS} / \mathrm{cm}, \beta=2000 \mathrm{~cm}^{-1}$, $\mathrm{R}=9100 \Omega \mathrm{cm}^{2}, \mathrm{I}_{\mathrm{S}}=50 \mu \mathrm{A} / \mathrm{mm}^{3}$ and $\alpha=2 \pi / 3$ [12]. Space constants $\lambda_{j}$ for the various directions $\mathbf{j}=\mathrm{l}, \mathrm{t}, \mathrm{n}$, are also presented in Table 1 . 
Table 1: Conductivity data (in $\mathrm{mS} / \mathrm{cm}$ ) and space constants (in $\mathrm{mm}$ ) from the indicated studies.

\begin{tabular}{cccccccccc} 
Study & $g_{e l}$ & $g_{e t}$ & $g_{e n}$ & $g_{i l}$ & $g_{i t}$ & $g_{i n}$ & $\lambda_{l}$ & $\lambda_{t}$ & $\lambda_{n}$ \\
\hline Nominal [25] & 2.6 & 1.04 & - & 2.6 & 0.26 & - & 0.77 & 0.31 & - \\
Hooks et al. [8] & 2.63 & 2.45 & 1.087 & 2.63 & 0.263 & 0.08 & 0.77 & 0.33 & 0.18
\end{tabular}

\section{Methods}

\subsection{Previous method for retrieving conductivities}

Johnston et al. [12] demonstrated that a particular electrode array, along with the inversion method discussed therein, successfully retrieves the four conductivities $g_{i l}, g_{i t}, g_{e l}$ and $g_{e t}$, as well as the fibre rotation angle $\alpha$, from measurements of potential. That array consists of 34 electrodes lying in two parallel planes on 17 micro needles (Figure 2). The array is aligned with the longitudinal and transverse fibre directions. The dimensions of the array given in Figure 2 were chosen because the potential measurements required to obtain the conductivities need to be made at distances less than or equal to the space constant of the tissue (referred to as closely spaced), as well as at distances greater than the space constant (referred to as widely spaced) [19]. Johnston et al. [12] found that that a two pass protocol is required to find the desired parameters accurately. On the first pass, potentials are stimulated and measured on a subset of electrodes that are closely spaced $(0.5 \mathrm{~mm})$ to give the extracellular conductivities [19]. These closely spaced electrodes are shown in plan view in Figure 3(a), along with the source and sink electrodes. Widely spaced electrodes $(1 \mathrm{~mm})$ are used in the second pass, with the extracellular conductivities found in the first pass held constant, to obtain the intracellular conductivities [19] and the fibre rotation angle. These widely spaced electrodes are shown in Figure 3(b). 


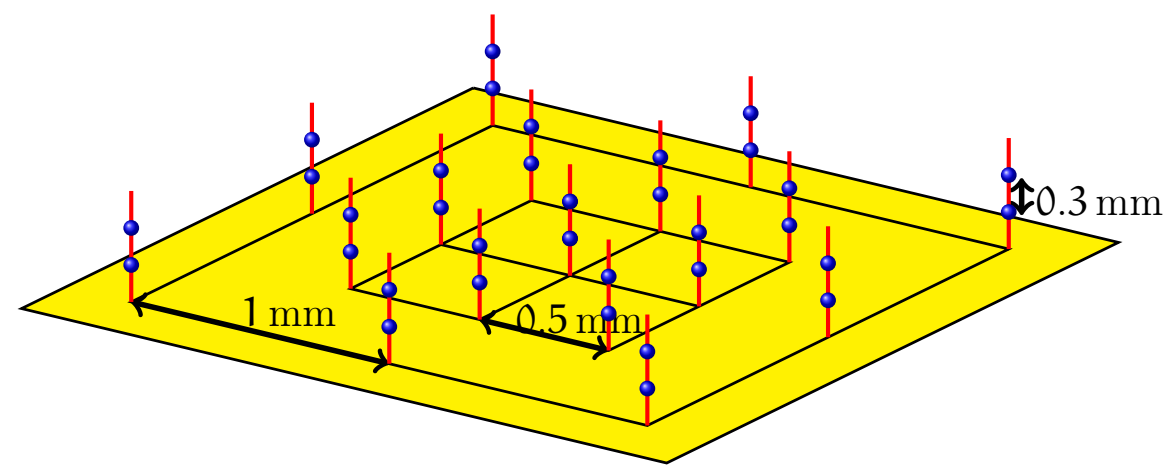

Figure 2: Previously proposed [12] two layer multi-electrode array used to retrieve four conductivities. The array consists of 17 micro needles, each containing two electrodes. Subsets of the electrodes are used in the two pass protocol (see Figure 3).

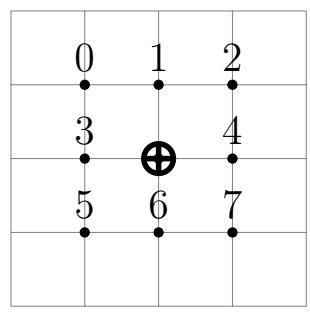

$z=0.3 \mathrm{~mm}$

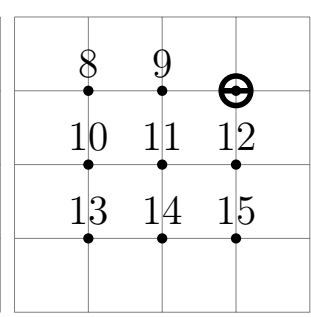

$z=0.6 \mathrm{~mm}$

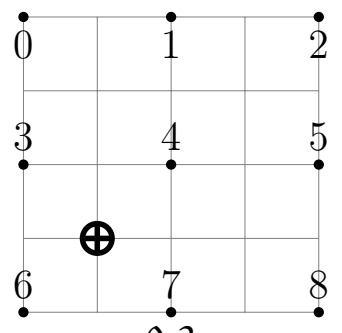

$z=0.3 \mathrm{~mm}$

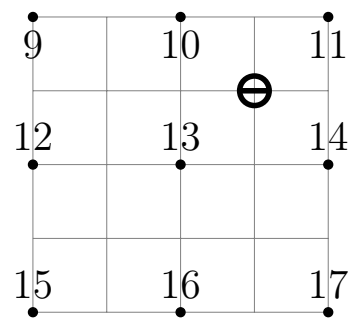

$z=0.6 \mathrm{~mm}$

(a) closely spaced electrode set

(b) widely spaced electrode set

Figure 3: Plan view of the two layers of the multi-electrode array, shown in Figure 2, that are used in (a) the first and (b) the second pass of the protocol to retrieve the four conductivities. Source and sink electrodes are indicated by + and - , respectively. 


\subsection{Sensitivity analysis}

To design an electrode array that will be able to retrieve six conductivities, rather than four, a sensitivity analysis is carried out on a variety of arrays. This is achieved by considering the sensitivity of the forward problem (no inversion involved) to variability in the conductivities. Specifically, $10 \%$ error is added to one conductivity value and the potential on the set of electrodes is compared with the potential when no change is made to the conductivity. Sensitivity is defined as the percentage relative error in $\phi_{e}$ at a particular electrode,

$$
\text { sensitivity }=\left|\frac{\phi_{e}-\phi_{e c}}{\phi_{e}}\right| \times 100 \%,
$$

where $\phi_{e}$ is the simulated potential at a particular electrode and $\phi_{e c}$ is the simulated potential at the same electrode, after $10 \%$ error is added to a conductivity value.

\section{Results and discussion}

\subsection{Sensitivity analysis on the original 2D array}

A sensitivity analysis is performed investigating the effect of variability in each of the four conductivity values, $g_{i l}, g_{i t}, g_{e l}, g_{e t}$, on the potentials measured by the electrodes. This is conducted on each of the closely spaced and widely spaced electrode sets of the original 2D array, described in Section 3.1. The sensitivity of each electrode to each of the four nominal conductivity values is shown in Figure 4 for (a) the closely spaced and (b) the widely spaced electrode sets. In each case one particularly sensitive electrode is identified (\#12 for case (a) and \#4 for case (b), with identifying numbers shown in Figure 3), along with one or two moderately sensitive electrodes. In case (a) 
the electrodes are sensitive to the extracellular conductivities and in case (b) to both the extracellular and the intracellular conductivities.

Additional sensitivity experiments were carried out where error was added to more than one conductivity value at a time but, due to the near linearity of most of the responses, it was decided to restrict the analysis to simply adding error individually to the conductivity values. The only electrodes for which the response was nonlinear were those that were particularly sensitive to adding error individually, and in those cases the sensitivity response was magnified.

Initially, it was not clear whether having a particularly sensitive electrode in a set was an advantage or a disadvantage, but studies into the effect of removing a sensitive electrode from a set indicated that electrode sets perform best, in terms of conductivity retrievals, when they contain at least one particularly sensitive electrode. This fact will now be used in analysing various possible arrays to see whether they may be suitable for retrieving six conductivity values.

\subsection{Sensitivity analysis for a 3D array}

Here we consider a 3D array that is an extension of the successful 2D array.

\subsubsection{Closely spaced electrode set in a 3D array}

The closely spaced electrode set is the same as the 2D version in Figure 3(a), except that it now has a third layer of electrodes (\#16-24) with spacing $0.5 \mathrm{~mm}$ in the normal direction. The sensitivity of these electrodes to changes in the conductivity values is plotted, for the data of Hooks et al. [8], in Figure 5(a) (note the different scale from Figure 4). The extracellular sensitivity of the most sensitive electrode \#11 is quite high for $g_{e l}$ and particularly so for $g_{e n}$, but not for $g_{e t}$. However, testing with the first pass inversion process showed 


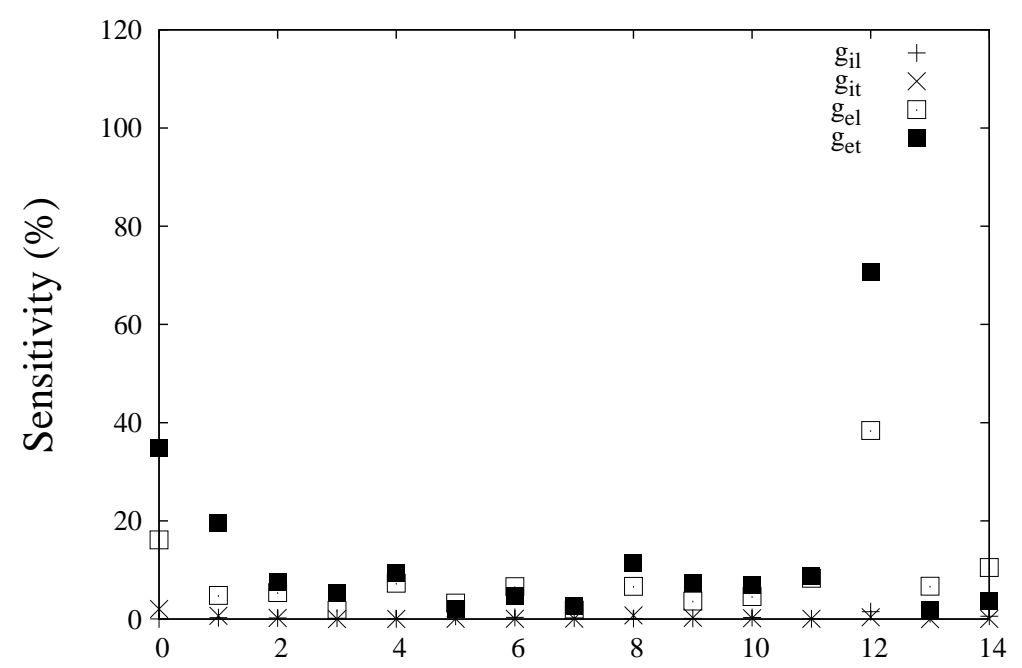

(a)

Electrode number
(b)

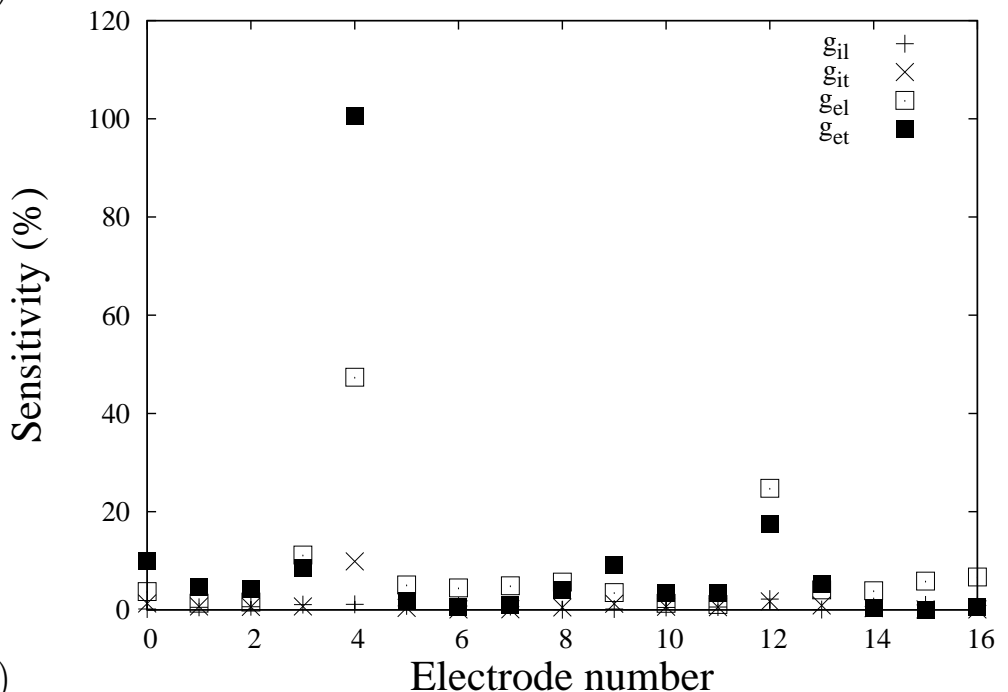

Figure 4: Sensitivity (percentage relative error in $\phi_{e}$ ) when $10 \%$ error is added to the conductivity values, for the electrodes used in (a) the first pass and (b) the second pass of the retrieval protocol for the four nominal conductivities [25]. The electrode arrays are shown in Figures 2 and 3. 
that this array works quite satisfactorily, perhaps because in this case there is a second electrode $(\# 16)$ that is quite sensitive to $\mathrm{g}_{\mathrm{et}}$.

\subsubsection{Widely spaced electrode sets in a 3D array}

The 3D extension of the widely spaced electrode set of Figure 3(b) with a third layer of electrodes $(\# 18-26)$ was tested for its sensitivity to the six conductivity values, but this set was not able to accurately retrieve the intracellular conductivities. Therefore, an alternative electrode set is proposed for the second pass.

The alternative widely spaced electrode set in Figure 6 contains 37 rather than 27 electrodes, resulting in both $0.5 \mathrm{~mm}$ and $1 \mathrm{~mm}$ electrode spacings in the longitudinal and transverse directions. The source and sink are also adjusted to be further apart. This new grid has a much higher sensitivity (Figure 5(b)) on its most sensitive electrode (\#17) than the 27 electrode set, with mean extracellular and intracellular values of 105 and 11, compared with 49 and 4 . Testing indicates that this array accurately retrieves the intracellular conductivity values.

\subsection{Array design}

The above work showned that it is possible to design an electrode array that can retrieve the six conductivity values of Hooks et al. [8] from measurements of potential. Later work (not presented) also shows that this is possible for the other available six conductivity dataset, that of MacLachlan et al. [18]. An additional study (not presented) indicates that, for both of these datasets, particularly sensitive electrodes are those with the lowest values of $\phi_{e}$.

This provides the basis for a technique that will allow the array design to be refined. Such a design needs to contain at least one electrode that is particularly sensitive to changes in the conductivity values. However, that 


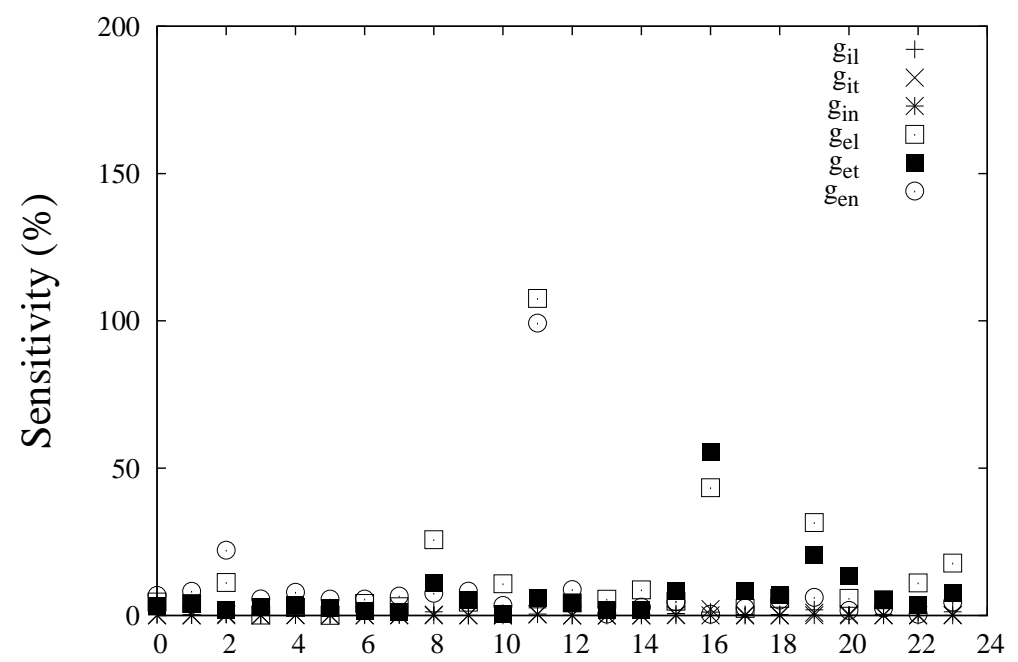

(a)
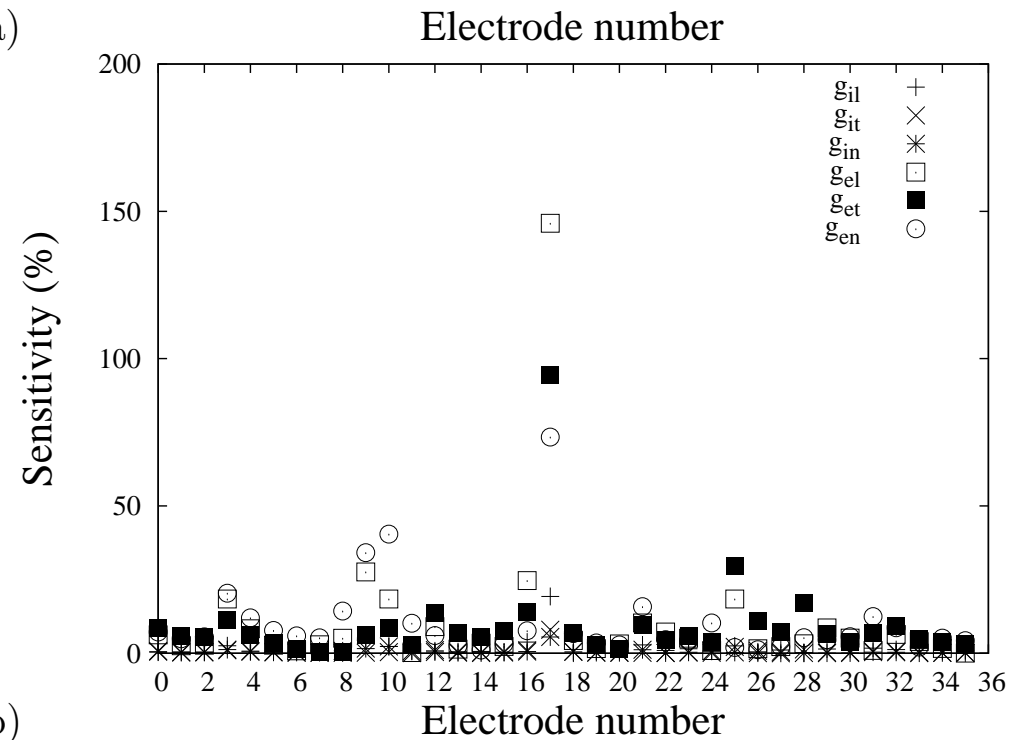

Figure 5: Sensitivity (percentage relative error in $\phi_{e}$ ), when 10\% error is added to the conductivity values, for the electrodes used in (a) the first pass and (b) the second pass of the retrieval protocol for the Hooks et al. [8] conductivities. The electrode set used for (a) is the same as Figure 3(a) except with a third layer of electrodes, and the electrode set used for (b) is shown in Figure 6. Here, the vertical scale is not the same as Figure 4. 

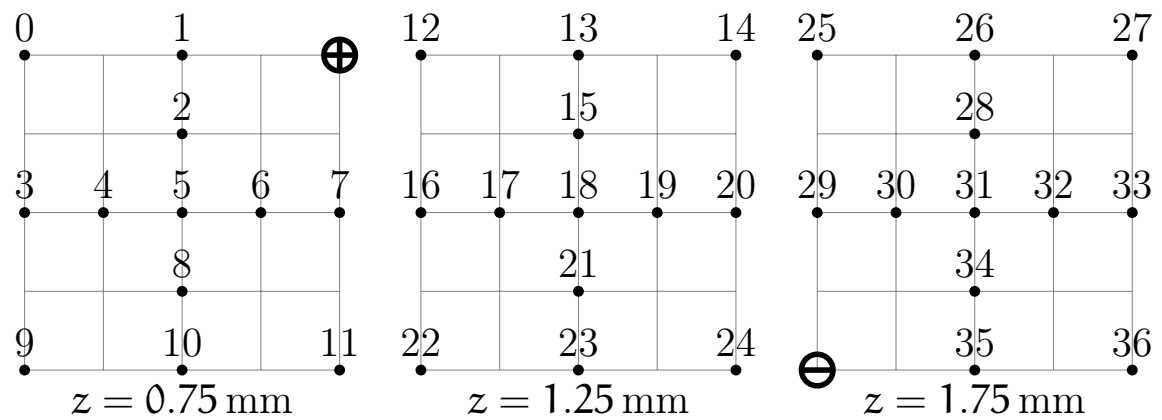

Figure 6: Plan view of the three layers of 37 widely spaced electrodes used in the second pass with the six conductivities of Hooks et al. [8]. Source and sink electrodes are indicated by + and - , respectively.

the conductivities are to be determined in this process, rather than known beforehand, would seem to mitigate against using an optimisation technique to determine the 'best' placement of the electrodes, since 'best' would have to apply to any possible sets of cardiac conductivity values, not just the two sets, from Hooks et al. [8] and MacLachlan et al. [18], that were used here for sensitivity testing.

\section{Conclusions}

As part of an effort to design a multi-electrode array capable of retrieving six conductivity values from measurements of potential, this study investigated the sensitivity of various arrays to changes in the conductivities in the model. Two types of electrode sets were studied: those that are closely spaced, which are used to retrieve the extracellular conductivities in the first pass of the inversion procedure; and those that are widely spaced and are used to retrieve the intracellular conductivities and fibre rotation angle in the second pass. By studying the sensitivity (percentage relative error in $\phi_{e}$ ) of the various 
electrodes, it was established that sets of electrodes that successfully retrieve the conductivities contain at least one particularly sensitive electrode. This fact was used to propose and test various $3 \mathrm{D}$ analogues of the previously published 2D array shown in Figure 2.

An analysis of the electrodes that are particularly sensitive reveals that these electrodes are the ones with the lowest values of $\phi_{e}$. This may then be the basis of a technique for analysing this and other electrode array designs using any other six conductivity datasets. Factors such as the placement of the source and sink electrodes, electrode spacing, the optimal number of electrodes and other such design features would then be able to be studied to ensure that such an array is capable of retrieving a wide enough range of values to include the 'true' conductivity values.

\section{References}

[1] R. M. Arthur and D. B. Geselowitz. Effect of inhomogeneities on the apparent location and magnitude of a cardiac current dipole source. IEEE Trans. Biomed. Eng., 17:141-146, 1970. doi:10.1109/TBME.1970.4502713 C273

[2] R. C. Barr and R. Plonsey. Electrode systems for measuring cardiac impedances using optical transmembrane potential sensors and interstitial electrodes-theoretical design. IEEE Transactions on Biomedical Engineering, 50(8):925-934, 2003. doi:10.1109/TBME.2003.814529 C273

[3] Bryan J. Caldwell, Mark L. Trew, Gregory B. Sands, Darren A. Hooks, Ian J. LeGrice, and Bruce H. Smaill. Three distinct directions of intramural activation reveal nonuniform side-to-side electrical coupling of ventricular myocytes. Circulation: Arrhythmia and Electropysiology, 2:433-440, 2009. doi:10.1161/CIRCEP.108.830133 C273 
[4] R. H. Clayton, O. Bernus, E. M. Cherry, H. Dierckx, F. H. Fenton, L. Mirabella, A. V. Panfilov, F. B. Sachse, G. Seemann, and H. Zhang. Models of cardiac tissue electrophysiology: Progress, challenges and open questions. Progress in Biophysics and Molecular Biology, 104(1-3):22-48, 2011. doi:10.1016/j.pbiomolbio.2010.05.008 C273

[5] L. Clerc. Directional differences of impulse spread in trabecular muscle from mammalian heart. Journal of Physiology, 255:335-346, 1976. http://www.ncbi.nlm.nih.gov/pmc/articles/PMC1309251/ C273

[6] Eliad Gilboa, Patricio S. La Rosa and Arye Nehorai. Estimating electrical conductivity tensors of biological tissues using microelectrode arrays. Annals of Biomedical Engineering, 40(10):2140-2155, 2012. doi:10.1007/s10439-012-0581-9 C273

[7] D. A. Hooks and M. L. Trew. Construction and validation of a plunge electrode array for three-dimensional determination of conductivity in the heart. IEEE Transactions on Biomedical Engineering, 55(2):626-635, 2008. doi:10.1109/TBME.2007.903705 C273

[8] Darren Hooks. Myocardial segment-specific model generation for simulating the electrical action of the heart. BioMedical Engineering OnLine, 6(1):21-21, 2007. doi:10.1186/1475-925X-6-21 C273, C274, C276, C277, C280, C282, C283, C284

[9] Darren A. Hooks, Karl A. Tomlinson, Scott G. Marsden, Ian J. LeGrice, Bruce H. Smaill, Andrew J. Pullan, and Peter J. Hunter. Cardiac microstructure: Implications for electrical propagation and defibrillation in the heart. Circulation Research, 91(4):331-338, 82002. doi:10.1161/01.RES.0000031957.70034.89 C273, C274

[10] Darren A. Hooks, Mark L. Trew, Bryan J. Caldwell, Gregory B. Sands, Ian J. LeGrice, and Bruce H. Smaill. Laminar arrangement of ventricular myocytes influences electrical behavior of the heart. Circulation Research, 101(10):e103-112, 2007. doi:10.1161/CIRCRESAHA.107.161075 C273 
[11] Barbara M. Johnston and Peter R. Johnston. Possible four-electrode configurations for measuring cardiac tissue fibre rotation. IEEE Transactions on Biomedical Engineering, 54(3):547-550, 2007. doi:10.1109/TBME.2006.890511 C274

[12] Barbara M. Johnston, Peter R. Johnston, and David Kilpatrick. Analysis of electrode configurations for measuring cardiac tissue conductivities and fibre rotation. Annals of Biomedical Engineering, 34(6):986-996, June 2006. doi:10.1007/s10439-006-9098-4 C273, C274, C276, C277, C278

[13] Barbara M. Johnston, Peter R. Johnston, and David Kilpatrick. A new approach to the determinination of cardiac potential distributions: Application to the analysis of electrode configurations. Mathematical Biosciences, 202(2):288-309, 2006. doi:10.1016/j.mbs.2006.04.004 C273, C274, C275, C276

[14] Barbara M. Johnston, Peter R. Johnston, and David Kilpatrick. A solution method for the determination of cardiac potential distributions with an alternating current source. Computer Methods in Biomechanics and Biomedical Engineering, 11(3):223-233, 2008. doi:10.1080/10255840701747594 C273

[15] Peter R. Johnston. Cardiac conductivity values-A challenge for experimentalists? Noninvasive Functional Source Imaging of the Brain and Heart 852011 8th International Conference on Bioelectromagnetism (NFSI \& ICBEM), pages 39-43, 13-16 May 2011. doi:10.1109/NFSI.2011.5936816 C273

[16] P. Le Guyader, P. Savard, R. Guardo, L. Pouliot, F. Trelles, and M. Meunier. Myocardial impedance measurements with a modified four electrode technique. 16th IEEE-EMBS, pages 880-881, 1994. doi:10.1109/IEMBS.1994.415193 C273

[17] P. Le Guyader, F. Trelles, and P. Savard. Extracellular measurement of anisotropic bidomain myocardial conductivities. I. Theoretical analysis. 
Annals of Biomedical Engineering, 29:862-877, 2001. doi:10.1114/1.1408923 C273

[18] M. C. MacLachlan, J. Sundnes, and G. T. Lines. Simulation of ST segment changes during subendocardial ischemia using a realistic 3-D cardiac geometry. IEEE Transactions on Biomedical Engineering, 52(5):799-807, 2005. doi:10.1109/TBME.2005.844270 C274, C282, C284

[19] R. Plonsey and R. C. Barr. The four-electrode resistivity technique as applied to cardiac muscle. IEEE Transactions on Biomedical Engineering, 29(7):541-546, 1982. doi:10.1109/TBME.1982.324927 C273, $\mathrm{C} 277$

[20] A. E. Pollard and R. C. Barr. Cardiac microimpedance measurement in two-dimensional models using multisite interstitial stimulation. American Journal of Physiology-Heart and Circulatory Physiology, 290(5):H1976-H1987, 2006. doi:10.1152/ajpheart.01180.2005 C273

[21] A. E. Pollard, C. D. Ellis, and W. M. Smith. Linear electrode arrays for stimulation and recording within cardiac tissue space constants. Biomedical Engineering, IEEE Transactions on, 55(4):1408-1414, 2008. doi:10.1109/TBME.2007.912401 C273

[22] Andrew E. Pollard and Roger C. Barr. A biophysical model for cardiac microimpedance measurements. American Journal of Physiology-Heart and Circulatory Physiology, 298:H1699-H1709, 2010. doi:10.1152/ajpheart.01131.2009 C273

[23] D. E. Roberts, L. T. Hersh, and A. M. Scher. Influence of cardiac fiber orientation on wavefront voltage, conduction velocity and tissue resistivity in the dog. Circ. Res., 44:701-712, 1979. doi:10.1161/01.RES.44.5.701 C273

[24] D. E. Roberts and A. M. Scher. Effects of tissue anisotropy on extracellular potential fields in canine myocardium in situ. Circ. Res., 50:342-351, 1982. doi:10.1161/01.RES.50.3.342 C273 
[25] B. J. Roth. Electrical conductivity values used with the bidomain model of cardiac tissue. IEEE Transactions on Biomedical Engineering, 44(4):326-328, April 1997. doi:10.1109/10.563303 C273, C276, C277, $\mathrm{C} 281$

[26] S. Rush, J. A. Abildskov, and R. McFee. Resistivity of body tissues at low frequencies. Circulation Research, 12:40-50, 1963. doi:10.1161/01.RES.12.1.40 C273

[27] R. Sadleir and C. Henriquez. Estimation of cardiac bidomain parameters from extracellular measurement: Two dimensional study. Annals of Biomedical Engineering, 34(8):1289-1303, 2006. doi:10.1007/s10439-006-9128-2 C273

[28] O. H. Schmitt. Biological information processing using the concept of interpenetrating domains. In K. N. Leibovic, editor, Information Processing in the Nervous System, chapter 18, pages 325-331. Springer-Verlag, New York, 1969. C275

[29] Kirsten H. W. J. Ten Tusscher, R. Hren, and A. V. Panfilov. Organization of ventricular fibrillation in the human heart. Circulation Research, 100(12):87-101, 2007. doi:10.1161/CIRCRESAHA.107.150730 $\mathrm{C} 273$

[30] Mark L. Trew, Bryan J. Caldwell, Thiranja P. Barbarenda Gamage, Gregory B. Sands, and Bruce H. Smaill. Experiment-specific models of ventricular electrical activation: Construction and application. In 30th Annual International IEEE EMBS Conference, pages 137-140, 2008. doi:10.1109/IEMBS.2008.4649109 C273

[31] L. Tung. A Bi-domain model for describing ischaemic myocardial DC potentials. PhD thesis, Massachusetts Institute of Technology, June 1978. http://hdl.handle.net/1721.1/16177 C273

[32] Mikael Wallman, Nicolas P. Smith, and Blanca Rodriguez. A comparative study of graph-based, eikonal, and monodomain 
simulations for the estimation of cardiac activation times. IEEE Transactions in Biomedical Engineering, 59(6):1739-1748, 2012. doi:10.1109/TBME.2012.2193398 C273

\section{Author address}

1. Barbara M. Johnston, School of Biomolecular and Physical Science, Griffith University, Nathan, Queensland 4111, Australia.

mailto:Barbara. Johnston@griffith. edu . au 